\section{Accessing the PrEP population: what is the best service model?}

\section{J. Shaw, L. Dufaur, J. Bayley}

Barts Health NHS Trust

\title{
Background:
}

Pre-Exposure Prophylaxis (PrEP) is effective to prevent HIV infections ${ }^{1}$. Optimal service models for delivering this intervention are as yet unclear. Our dedicated East One clinic provides risk assessment, advice and monitoring for privately sourced PrEP as well as psychological and practical support for 'chemsex' ( recreational drug use in sexualised context) and STI screening for high risk MSM.

\section{Aims:}

To identify and characterise the patients attending the EastOne Clinic, specifically those taking PrEP, regarding sexual behaviour, drug use and STI rates, in order to improve the service provided.

\section{Methods:}

A retrospective electronic case note review was conducted for patients attending clinic from January - November 2016. Data was collected on demographics (age, ethnicity, country of birth); sexual behaviour, "chemsex and STI prevalence. Data was collected for dosing of PrEP (daily dosing or event-based) and therapeutic monitoring of PrEP including renal function blood and urine tests. Data was analysed with STATA.

\section{Results:}

- A total of 54 male patients attended a total of 116 visits, with a median age of 42 years (IQR $32-44.5)$.

- $90 \%$ were white ethnicity (figure 1 ). All had sex only with men.

- Of these 54 patients, only 27 (50\%) started PrEP and engaged in care.

- Median Number of partners was 4 partners in the preceding 90 days with no difference whether on or off PrEP. Result much lower than encountered in the PROUD trial (See table 1 for comparison with PROUD study results).

- For our population, there were lower than expected rates of STI' s: 6\% for $\mathrm{CT}$ and $9 \%$ for GC - total for any site: rectal, throat or urethral.

- No positive results for HCV Ab, HBsAg or HIV.

- $39 \%(21 / 54)$ had used 'chems' at some point, with $19 \%(4 / 21)$ of those reported 'slamming' (intravenous use) in the last 3 months.

- Routine urinalysis showed $30 \%$ abnormalities (i.e trace protein or +1 protein), but no subsequent abnormal uPCR results were found, despite $12 \%$ patients reporting dietary protein supplements.

\section{Conclusion:}

As the interest and use of PrEP grows, new service models may have to be developed to accommodate this population. We found varying levels of engagement with patients who were predominantly white, older, and relatively low sexual-risk. Local factors such as a fixed time-slot on Monday afternoons from 1630-1830 and use of booked appointments rather than walk-in may contribute to low patient numbers. The regular monitoring required for PrEP use and the current necessity for self-sourcing PrEP in England may be an inherent deterrent for use amongst younger patients or patients with a chaotic lifestyle.

Engagement with BME and MSM communities, along with drug services, may be needed to inform effective delivery of this intervention to those most at risk.

\section{References:}

1) Dolling et al. Trials (2016) 17:163 An analysis of baseline data from the PROUD study: an open-label randomised trial of pre-exposure prophylaxis.

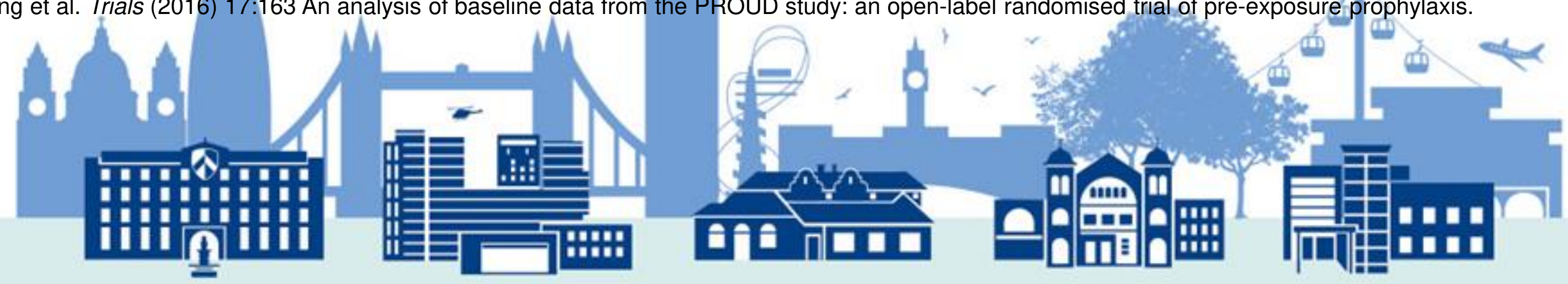

